\title{
Research Progresses of the Mechanism of Insulin Resistance in Type II Diabetes
}

\author{
Xinming Liu, \\ ${ }^{1}$ Biotechnology, Department of Biochemistry and Biotechnology, Xiamen University, 4221-120 Xiang'an South Road, Xiamen, China
}

\begin{abstract}
Insulin resistance(IR) is the most primary pathological mechanism of type II diabetes, a metabolic disorder characterized by chronic hyperglycemia. Insulin transduction pathways have significant effects on IR, and they are mainly classified into two pathways: insulin receptor substrate-1 (IRS1)/phosphatidyl inositol 3 kinase (PI3K)/protein kinase (PKB) pathway and Ras/Raf/mitogen-activated protein kinase (MAPK) pathway. Resulted from various reasons such as obesity, oxidative stress and endoplasmic reticulum(ER) stress, if any site in the pathway is abnormal, IR will be caused. Therapies to IR is to enhance insulin sensitization, including adiponectin, endolipid and metformin. We review the mechanisms of IR in type II diabetes.
\end{abstract}

\section{Introduction}

Diabetes mellitus (DM) is a metabolic disease featured by high blood glucose as a consequence of defective insulin secretion, impaired insulin functions or both. DM is distributed into four categories, type I, type II, gestational diabetes, and other specific types.[1] The feature of type I is lack of insulin resulted from damaged pancreatic beta cells or is autoimmune by antibodies, while the features of type II are not short of insulin but target cells are not sensitive to insulin.

IR is the most important mechanism of type II DM. The abnormal insulin signalling pathway has effect on the occurrence and development of IR. Common insulin signalling pathways include the PI3K pathway and MAPK pathway.[2] After the combination of insulin and insulin receptor, the dysfunction of signal transduction process includes decreased activity of tyrosine kinase of insulin receptors, abnormal insulin signal transduction, reduced glucose transport, decreased glucose phosphorylation and decreased activity of glycogen synthase. The full attention and in-depth understanding of dysfunctional insulin signalling and IR caused by have important guiding significance to study the nosogenesis of diabetes and the progress of targeted hypoglycemic drugs.

\section{Two Pathways of Insulin Signalling Transduction}

Insulin binds to receptors on the surface of target tissues such as liver, adipose tissue and skeletal muscle, which triggers phosphorylation and excitation of receptor tyrosine kinase (RTK). A cascade occurs and produces multiple biological effects. There are two pathways,
IRS-1/PI3K/protein kinase pathway and Ras/Raf/MAPK pathway.[2]

In the first pathway, exogenous insulin or insulin secreted by beta cells get to target tissues, bind to insulin receptor $\alpha$ subunits and remove inhibition of $\beta$ subunits. The activity of $\beta$ subunits on PTK are activated and their phosphorylation abilities are increased which not only self-phosphorylated and also phosphorylate the tyrosine sites on IRS-1. [3] Activated IRS-1 binds to PI3K and arches to target cell membrane.[4, 5] The activated PI3K phosphorylates phosphatidylinositol (PI) and generates phosphatidylinositol 2 phosphate (PIP2) and phosphatidylinositol 3 phosphate (PIP3). PIP3 triggers multiple mechanisms to transfer signals and excites protein kinase $\mathrm{B}$ (PKB), which is also named AKT. [6] On the one hand, activated PKB inactivates the Ser sites of glycogen synthase kinase-3 (GSK-3) by phosphorylation and thereby activates glycogen synthase which promotes the synthesis of glycogen.[7] On the other hand, activated PKB enhances the activity of glucose transporter 4 (GLUT4) and thus enhancing glucose ingestion of liver cells, skeletal muscle cells and adipose cells.

In the second pathway, after insulin activates receptors through the above approach, phosphorylated insulin receptors activate IRS-2. Activated tyrosine sites on IRS-2 binds to $\mathrm{SH} 2$ domain of growth factor receptor-bound protein 2 (Grb2) and consequently interacts with guanosine diphosphate (GDP)/guanosine5'-triphosphate(GTP) transfer factor, which transforms inactivated Ras-GDP into activated Ras-GTP and thus activating Ras.[8] Ras is GTP-binding protein which has great significance in signalling transduction. Otherwise, activated insulin receptors can also activate Ras through another way. Insulin receptors interact with signal protein Shc and tyrosine phosphates Shc. Activated Shc

\footnotetext{
*Corresponding author: author@e-mail.org
} 
transfers the signal to Grb2 and activates GDP/GTP transfer factor and consequently activates Ras.[9, 10] Activated Ras recruits and excites Raf serine kinase which serine phosphorylates mitogen-activated protein kinase kinase (MAPKK) and activates MAPK. MAPK activation can induce phosphorylation of many target protein and accordingly participate in the phosphorylation progress of some transcription factors, regulation of gene transcription and apoptosis.[11] (fig. 1)

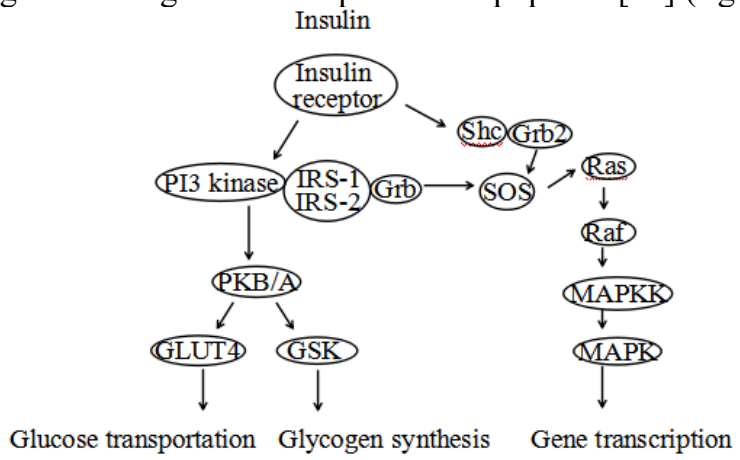

Fig. 1. Insulin transduction for insulin receptor. After insulin binding to receptors, cascade reactions occur and are divided into two pathways. In IRS-1/PI3K/PKB pathway, GSK3 and GLUT4 are finally activated. In Ras/Raf/MAPK pathway, MAPK is phosphorylated and regulates gene expression. " $\rightarrow$ " means simulative signalling transduction, "SOS" means son of sevenless.

\section{Factors for the formation of IR}

\subsection{Abnormities in Insulin Signalling Pathway}

\subsubsection{IRS Abnormities}

IRS family are important adaptor protein in insulin signalling pathway. They can be tyrosine phosphorylated by activated insulin receptor and then transduce signal downward. The abnormities of IRS mainly reflect in the following aspects: (1)The abnormal degradation of IRS contributes to the decrease of its content and of the sensibility of the target cell to insulin.[12] (2)The abnormal phosphorylation of IRS, including the abnormal increased phosphorylation level of serine or threonine site and the decreased phosphorylation level of tyrosine site.[13, 14] (3)The abnormal distribution of IRS. IRS aggregates in the cytoplasm in excess and the number of correctly oriented IRS on the cytoskeleton decreases, which finally causes IR.[15]

\subsubsection{PI3K Abnormities}

The decrease of expression or activity of PI3K leads to the inability of insulin signalling transfer through PI3K pathway and consequently causing IR.[16, 17]

\subsubsection{PKB/AKT Abnormities}

$\mathrm{PKB} / \mathrm{AKT}$ is the direct target protein of PI3K. The decreased expression or activity of PI3K can result in the formation of IR.[16, 17]

\subsubsection{GSK Abnormities}

Under the influence of insulin, GSK-3 is inactivated by phosphorylation and the synthesis of glycogen is initiated, which promotes the transportation of glucose. The increase of GSK-3 expression level is closely related to the occur and development of IR.[16, 17]

\subsubsection{GLUT4 Abnormities}

The decreased expression, transposition obstacle and decreased inner activity of GLUT4 bring about the reduction of glucose uptake and impaired metabolism and thus obstacle in insulin function. $[16,17]$

\subsection{Adipocyte Factors}

Adipocyte factors, including tumor necrosis factor (TNF), resistin (RSTN) and free fatty acid (FFA), can induce unspecific chronic inflammation of target tissue, disturb insulin signalling pathway and cause IR. In addition, other adipocyte factors like adiponectin (ADPN) and visfatin (VF) can improve response state of IR inflammation by upregulation of related protein activity in insulin signalling pathway.[18]

\subsubsection{Pro-inflammatory factors}

The macrophages in adipose tissue secrete TNF- $\alpha$, interleukin(IL)- 6 and IL-1 $\beta$, which activate inflammation signals in insulin target cells, activate C-Jun N-terminal kinases (JNK) and inflammation transcription factors activator protein 1 (AP-1) and nuclear factor kappalight-chain-enhancer of activated B cells (NF- $\kappa \mathrm{B})$ and then induce the generation of inflammation factors. Among them, JNK pathway phosphorylates insulin receptors and IRS and results in IR.[19] TNF- $\alpha$ accelerates the degradation of fat and the number of FFA in blood increases; inhibits expression of GLUT4 and thus suppressing glucose transportation induced by insulin. TNF $\alpha$, IL-6 and IL-1 can serine phosphorylates IRS-1, induces suppressor of cytokine signalling 3 (SOCS-3) generation and decreases IRS-1 expression; increases protein tyrosine phosphatase 1B (PTP1B) gene transcription in adipocytes and promotes the expression and secretion of inflammation factors including IL-6、 IL-8、IL-1ß.[20-23] In hepatic tissue, in the condition of steatohepatitis, inflammation signalling pathway is activated, and liver cells release cytokines to activate macrophage Kupffer cells and mediates IR.[24]

\subsubsection{Resistin}

Resistin is mainly secreted in white adipose tissue. (1) Resistin promoted the expression of socs-3 gene in adipocytes[25] and inhibits phosphorylation of tyrosine 
residues by competition binding[26] and thus restraining the activity of GLUT4 protein. (2)Resistin suppresses AMPK pathway. Insulin signal transduction is weakened, cyclic adenosine monophosphate (cAMP) concentration is increased, and AMPK starts metabolism, which promotes gluconeogenesis to increase blood glucose amount. Resistin reduces AMPK phosphorylation and AKT phosphorylation, which lead to IR.[27] (3)Resistin significantly promotes the degradation of triglycerides into fatty acids and glycerol, and increases FFA in serum.[28] (4)Resistin and inflammatory cytokines upregulate each other, relying on NF- $\mathrm{BB}$ pathway.[29] (5)Resistin and LPS competitively bind to toll-like receptor 4 (TLR4),[30] which activates JNK and p38, leads to inflammation and inhibits IRS-1 phosphorylation.[31] (6)Resistin increases the level of PTP1B, a protein that promotes IR.[31]

\subsubsection{Free fatty acid}

Plentiful studies have shown that saturated FFA reduces the sensitivity of target tissue cells to insulin and reduces their use of glucose. FFA decreases the expression and activity of insulin receptors on different types of muscle cells. Meanwhile, the phosphorylation of IRS-1, IRS-2, PKB and GSK3 are inhibited, which impedes the insulin signal transduction of PKB pathway.[32] FFA can also activate inhibitor of nuclear factor kappa-B kinase subunit beta (IKK)/NF-kB inflammatory signalling pathway and JNK pathway, resulting in increased serine/threonine phosphorylation level on IRS, decreased tyrosine phosphorylation and IRS activity, and promote the occurrence of IR.[33] Tremblay has found that FFA suppresses the amount of GLUT4 and reduces glucose transportation, leading to IR finally.[34]

\subsection{ER Stress}

Incorrect folded protein, volatile nutrients, toxins or virus infection can cause ER stress, which will in turn activate unfolded protein response (UPR) signal systems----mediated by inositol-requiring enzyme 1(IRE-1), protein kinase $\mathrm{R}$ (PKR)-like endoplasmic reticulum kinase(PERK), ATF6 and cause ER adaptive response. Among them, IRE-1 and PERK activation can initiate two important inflammatory pathways, namely JNK and IKK $\beta$.[35] High concentration of inflammatory molecules and saturated FFAs can induce ER stress and IR.[36]

\subsection{Oxidative Stress}

ER stress and induce mitochondrial dysfunction reduced nicotinamide adenine dinucleotide phosphate (NADPH) oxidase and inductive nitric oxide synthase (iNOS) make obese fat cells produce a large amount of active oxygen ROS, which initiates systematic oxidative stress. High concentrations of plasma FFAs and inflammatory molecules can both promote the expression and activity of the two oxidases in fat tissue.[37] Oxidative stress activates JNK and IKK $\beta$ directly which serine phosphorylates IRS-1,[38] and can also stimulate the secretion from fat tissue of inflammatory molecules such as IL -6 and TNF $\alpha .[39]$

\subsection{Leptin}

Leptin is the expression outcome of ob gene in fat tissue, which mainly reduces fat deposition of the body by inhibiting appetite, stimulate fat decomposition and heat generation. High levels of leptin by exciting Janus kinase (JAK) - signal transducers and activators of transcription 3 (STAT3) signal to promote the transcription of SOCS3, thereby inhibiting the expression of the IRS-1 in insulin signal. Leptin can also activate the ERK signal, serine phosphorylates IRS-1, and induce IR.[40]

\section{Therapies to enhance insulin sensitization}

\subsection{Adiponectin}

Adiponectin, a kind of cytokine secreted by adipocytes, contributes to binding to adiponectin receptors, and has the functions of regulating sugar and lipid metabolism, increasing the oxidation of fatty acids, increasing glucose uptake and improving insulin sensitivity.[41] Adiponectin receptor 1 can enhance the regulation of adiponectin on AMPK[42], p38MAPK and peroxisome proliferator activated receptor $\alpha$, promote the oxidation of fatty acids, increase the ingestion of glucose and inhibit the synthesis of glycogen, reduce the lipid content in cells and improve the sensitivity to insulin.[43] Adiponectin can inhibit the lipid hydrolysis by TNF $\alpha$ and reduce the amount of free fatty acids in blood system.[44] Adiponectin increases tyrosine phosphorylation extent of insulin receptors, IRS-1, IRS-2, and AKT in the PI3K/AKT pathway, which promotes insulin signal transduction.[45]

\subsection{Endolipid}

Endolipid can also significantly increase tyrosine phosphorylation level of IRS-1, IRS-2, and AKT,[46] up-regulate the expression of PI3K and GLUT4, increase glucose uptake,[47] and activate the activity of MAPK. Endolipid improve IR by facilitating insulin signal transduction in two important pathways: MAPK and PI3K.[48]

\subsection{Rosiglitazone}

A possible mechanism is related to the inhibition of the expression of $\mathrm{TNF} \alpha$. Rosiglitazone can significantly inhibit TNF-production in adipose tissue and promote the expression of GLUT4 in skeletal muscle[49], thereby improving the body's sensitivity to insulin. 


\subsection{Metformin}

Metformin activates AMPK pathway, inhibits transforming growth factor beta 1 (TGF $\beta 1$ ) pathway, inhibits fatty tissue fibrosis and finally improves IR.[50]

\section{Conclusion}

Insulin signal transduction is a process in which insulin signals are transmitted step by step and amplified in target tissue cells through a series of phosphorylation cascade to produce biological effects. Abnormal insulin signal transduction units or multiple sites caused by various factors, such as insulin degradation enzyme gene, protein tyrosine phosphatase, proteasome, fat factor, oxidative stress and endoplasmic reticulum stress, can all contribute to IR. The key point of therapy is to promote the sensitization of insulin to target cells. Researchers have found that adiponectin, endolipid, rosiglitazone and metformin play a significant role in this part.

However, at present, there are many mechanisms that are not completely clear, such as the specific mechanism of signal transduction downstream of PI3K and MAPK, the role of pigment epithelium-derived factor (PEDF) in two pathways which is still controversial, and the effect of new found factors on insulin signal transduction pathways. On one hand, PEDF increases the phosphorylation of JNK and reduces the phosphorylation at active sites of IRS1 and Akt, resulting in IR.[51] On the other hand, some researches indicated that PEDF inhibits RAS-1 activity, IRS-1 tyrosine phosphorylation of stem cells, JNK activity and ROS production, improving IR.[52] The function of IL-6 in IR of adipocytes remains disputable. It is generally believed that IL-6 concentration in blood is positively related to obesity, glucose tolerance and IR.[53] However, studies have also concluded that IL-6 can enhance the glucose ingestion, glycogen produce and glucose oxidation of human skeletal muscle cells in resting state, and its mechanism is related to the increased phosphorylation of IL-6 in signal transducers and transcriptional activator 3(STAT3), AMPK and p38 MAPK. [54] Further research is needed on PEDF and IL-6 in the future.

Apart from these, microRNA is a spot worthy of further studying. Inhibition of mir-200 family expression significantly increased the levels of phosphatase and tensin homolog (PTEN) and Zinc finger protein 2 (ZFPM2). ZFPM2 also called transcriptional regulator friend of Gata 2 (FOG2). FOG2 competitively binds PI3K could impede insulin signal transduction, because of FOG2 could reduce the activity of PI3K and inhibit phosphorylation of PKB and GSK. PTEN catalyzes the downstream of PI3K to generate PIP2, blocks the signal pathway of PI3K downstream, reduces PKB activity and produces IR. By contrast, overexpression of mir-200 family inhibits FOG2 and PTEN, increases the phosphorylation level of PKB/GST, and improves IR.[55] It is significant to maintain high level of mir-200 family and scientists could develop relevant medicine.

Recently, diabetes is one of the most vital disease that pose a threat to human health. The number of patients is rising continually, thus the understanding of diabetes mechanism and therapy is urgent. In addition to medical treatment, lifestyle changes could also control diabetes. For instance, doing aerobic sports can improve the combination ability of insulin and insulin receptors.[56] Finally, there is still a long way for scientists and patients to go for the disease.

\section{References}

1. Shoback DG, Gardner D, et al, "Chapter 17". Greenspan's basic \& clinical endocrinology (9th ed.). New York: McGraw-Hill Medical (2011)

2. F.Y. Zhou, Y.F. Yang, Y. Shi, Advances in Insulin Resistance and its Signalling Pathway, J. Liaoning Univ. Tradit. Chin. Med. 18, 71-73 (2016)

3. M.N. Chi M, Y. Ye, X.D. Zhang, et al, Insulin induces drug resistance in melanoma through activation of the PI3K/AKT pathway, Drug Des., Dev. Ther. 2, 255-262 (2014)

4. Y.L. Wu, X.T. Chang, Interactions between Insulin Signalling Pathway, Inflammation Signalling Pathway and ubiquitin-proteasome system, Chin. J. Biochem. Mol. Biol. 32, 1177-1184 (2016)

5. Y. Zhang, X. Zhang, H. Zhang, et al, Kinase AKT controls innate immune cell development and function, Immunol. 140, 143-152 (2013)

6. J. Yang, T. Ikezoe, C. Nishioka, et al, Bcr-Abl activates AURKA and AURKB in chronic myeloid leukemia cells via AKT signalling, Int. J. Cancer, 134, 1183-1194 (2013)

7. X.K. Zheng, W.W. Wang, W.S. Feng, Advance in the study of the insulin-stimulated PI3K/PKB signal pathway, Chin. J. New Drugs, 21, 2763-2770 (2012)

8. C. De Luca, J.M. Olefsky, Inflammation and insulin resistance, FEBS Lett, 582, 97-105 (2008)

9. A. Belfiore, R. Malaguamera, Insulin receptor and cancer, Endocr.-Relat. Cancer. 18, 125-147 (2011)

10. R. Malaguamera, A. Belfiore, The emerging role of insulin and insulin-like growth factor signalling in cancer stem cells, Front. Cancer Endocrinol. 5, 10 (2014)

11. M.L. Goalstone, ERK 5: A Novel IKK $\alpha$-kinase in rat hippocampal neurons, Can. J. Neurol. Sci. 38, 639-648 (2011)

12. L. Rui, T.L. Fisher, J. Thomas, et al, Regulation of insulin/insulin-like growth factor-1 signalling by proteasome-mediated degradation of insulin receptor substrate-2, J. Biol. Chem. 276, $40362-40367$ (2001)

13. Z. Gao, X. Zhang, Zuberia, et al, Inhibition of insulin sensitivity by free fatty acids requires activation of multiple aerine kinases in 3T3-L1 adipocytes, Mol. Endocrinol. 18, 2024-2034 (2004)

14. T.Y. Choi, J.E. Kwon, E.S. Durrance, et al, Melatonin inhibits voltage-sensitive $\mathrm{Ca}(2+)$ channelmediated neurotransmitter release. Brain Res. 1557, $34-42$ (2014) 
15. L.A. Nolte, D.H. Han, P.A. Hansen, et al, A Peroxovanadium Compound Stimulates Muscle Glucose Transport as Powerfully As insulin and Contractions Combined, Diabetes, 52, 1918-1925 (2003)

16. H.Y. Zhao, Y. Wang, Y.P. Ma, et al, Insulin signal transduction disorder and insulin resistance, New Med. 41, 267-271 (2010)

17. J. Zhang, C.J. Zhao, H. Cai, The pathophysiological mechanisms of insulin resistance and relative cardiovascular injury, Anhui Medical and Pharmaceutical Journal, 22, 207-211 (2018)

18. Y.K. Wang, Advances in insulin signal transduction and insulin resistance, Hebei Medical University (2017)

19. J.M. Olefsky, C.K. Glass, Macrophages, inflammation, and insulin resistance. Annu. Rev. Physiol. 72, 219-246 (2010)

20. C.C. Chuang, K. Martinez, G. Xie, et al, Quercetin is equally or more effective than resveratrol in attenuating tumor necrosis factor-\{alpha\}-mediated inflammation and insulin resistance in primary human adipocytes. Am. J. Clin. Nutr. 92, 1511-1521 (2010)

21. I. Nieto-Vazquez, S. Fernández-Veledo, C. de Alvaro, et al, Dual role of interleukin-6 in regulating insulin sensitivity in murine skeletal muscle, Diabetes, 57, 3211-3221 (2008)

22. J. He, I. Usui, K. Ishizuka, et al. Interleukin-1alpha inhibits insulin signalling with phosphorylating insulin receptor substrate-1 on serine residues in 3T3-L1 adipocytes, Mol. Endocrinol. 20, 114-124 (2006)

23. T. Uno, J. He, Usui I, et al, Long-term interleukin1alpha treatment inhibits insulin signalling via IL-6 production and SOCS3 expression in 3T3-L1 adipocytes, Horm. Metab. Res. 40, 8-12 (2008)

24. D.Y. Oh, H. Morinaga, S. Talukdar, et al, Increased macrophage migration into adipose tissue in obese mice, Diabetes, 61, 346-354 (2012)

25. M. Pirvulescu, I. Manduteanu, A.M. Gan, D. Stan, V. Simion, E. Butoi, M. Calin, M. Simionescu, A novel pro-inflammatory mechanism of action of resistin in human endothelial cells: up-regulation of SOCS3 expression through STAT3 activation, Biochem. Biophys. Res. Commun. 422, 321-326 (2012)

26. C.M. Steppan, J. Wang, E.L. Whiteman, M.J. Birnbaum, M.A. Lazar, Activation of SOCS-3 by resistin, Mol. Cell Biol. 25, 1569-1575 (2005)

27. Y. REN, Z.C. Zuo, T.M. Wan, Resistin: It's role in insulin resistance and mechanism of action, Acta Physiologica Sinica, 2016, 68, 65-74 (2016)

28. C.L. Bai, D. Luo, Y.F. Liu, S.H. Chen, Y. Liu, The preparation of resistin mature peptide and its effect on glucose and fat metabolism in pigs. Prog. Veter. Med. 34, 46-50 (2013)
29. M. Bokarewa, I. Nagaev, L. Dahlberg, U. Smith, A. Tarkowski, Resistin, an adipokine with potent proinflammatory properties, J. Immunol. 174, 57895795 (2005)

30. A. Tarkowski, J. Bjersing, A. Shestakov, M.I. Bokarewa, Resistin competes with lipopolysaccharide for binding to toll-like receptor 4, J. Cell Mo.1 Med. 14, 1419-1431 (2010)

31. Tarkowski A, Bjersing J, Shestakov A, Bokarewa MI. Resistin competes with lipopolysaccharide for binding to tolllike receptor 4. J Cell Mol Med 2010; 14(6B): 1419-1431.

32. D. Dey, M. Mukherjee, D. Basu, et al, Inhibition of insulin receptor gene expression and insulin signalling by fatty acid: interplay of PKC isoforms therein, Cell Physiol. Biochem. 16, 217-228 (2005)

33. K. Paz, R. Hemi, D. LeRoith, et al, A molecular basis for insulin resistance. Elevated serine/threonine phosphorylation of IRS-1 and IRS2 inhibits their binding to the juxtamembrane region of the insulin receptor and impairs their ability to undergo insulin-induced throsine phosphorylation, J. Biol. Chem. 272, 29911-29918 (1997)

34. F. Tremblay, C. Lavigne, H. Jacques, et al, Defective insulin-induced GLUT4 translocation in skeletal muscle of high fat 2 fed rats is associated with alerstions in both $\mathrm{AKT} /$ protein kinase $\mathrm{B}$ and atypical kinase C activities, Diabetes, 50, 1901-1910 (2001)

35. Y.Q. Yang, M.Q. Yang, Inflammatory Mechanisms in Obesity-induced Insulin Resistance, Chinese J. Biochem. Mol. Biol. 28, 692-699 (2012)

36. M. Cnop, F. Foufelle, L.A. Velloso, Endoplasmic reticulum stress, obesity and diabetes, Trends Mol. Med. 18, 59-68 (2012)

37. J. Park, S.S. Choe, A.H. Choi, et al, Increase in glucose-6-phosphate dehydrogenase in adipocytes stimulates oxidative stress and inflammatory signals. Diabetes, 55, 2939-2949 (2006)

38. R. Vinayagamoorthi, Z. Bobby, M.G. Sridhar, Antioxidants preserve redox balance and inhibit cJun-N-terminal kinase pathway while improving insulin signalling in fat-fed rats: evidence for the role of oxidative stress on IRS-1 serine phosphorylation and insulin resistance, J Endocrinol, 197, 287-296 (2008)

39. S. Furukawa, T. Fujita, M. Shimabukuro, et al, Increased oxidative stress in obesity and its impact on metabolic syndrome, J. Clin. Invest. 114, 17521761 (2004)

40. C.J. Wang, Y. Zhang, Y.F. Guan, et al, Leptin and regulation of carbohydrate and lipid metabolism, Chin. J. Biochem. Mol. Biol. 25, 896-902 (2009)

41. W.X. Ding, Y.B. Dong, N. Ding, et al, Adiponectin protects rat heart from left ventricular remodeling induced by chronic intermittent hypoxia via inhibition of TGF- $\beta / \mathrm{smad} 2 / 3$ pathway, J. Thorac. Dis. 6, 1278-1284 (2014) 
42. T. Yamauchi, J. Kamon, H. Waki, et al, The fatderived hormone adiponectin reverses insulin resistance associated with both lipoatrophy and obesity, Nat. Med. 7, 941-946 (2001)

43. B.N. Finck, The role of the peroxisome proliferatoractivated receptor alpha pathway in pathological remodeling of the diabetic heart, Curr. Opin. Clin. Nutr. Metab. Care, 7, 391-396 (2004)

44. Q. Zhang, Y.J. Liu, L. Xu, et al, Influence of Adiponectin on Insulin Resistance and Ventricular Remodeling. Chinese General Practice, 19, 14011407 (2016)

45. M. Awazawa, K. Ueki, K. Inabe, et al, Adiponectin enhances insulin sensitivity by increasing hepatic IRS-2 expression via a macrophage-derived IL-6dependent pathway, Cell Metab. 13, 401-412 (2011)

46. A. Fukuhara, M. Matsuda, M. Nishizawa, et al, Visfatin: a protein secreted by visceral fa that mimics the effects of insulin, Science, 307, 426-430 (2005)

47. H.K. Song, M.H. Lee, B.K. Kim, et al, Visfatin: a new player in mesangial cell physiology and diabetic nephropathy, Am. J. Physiol-Renal, 295, F1485-F1494 (2008)

48. K. Schoonjans, G. Martin, B. Staels, et al, Peroxisome proliferator-activated receptors, orphans with ligands and functions, Curr. Opin. Lipidol. 8, 159-166 (1997)

49. G. Gurusubramanian, V.K. Roy, Expression of visfatin in alloxan-induced diabetic rat testis, Acta Histochem. 116, 1462-1468 (2014)

50. T. Luo, AMPK Activation by Metformin Suppresses Abnormal Adipose Tissue Extracellular Matrix Remodeling and Ameliorates Insulin Resistance in Obesity, Chongqing Med. Univ. (2016)

51. T. Yoshida, S. Yamagishi, K. Nakamura, et al, Pigment epithelium-derived factor (PEDF) ameliorates advanced glycation end product (AGE)induced hepatic insulin resistance in vitro by suppressing Rac-1 activation, Horm. Metab. Res. 40, 620-625 (2008)

52. S. Crowe, L. E. Wu, C. Economou, et al, Pigment Epithelium-Derived Factor Contributes to Insulin Resistance in Obesity, Cell Metab. 10, 40-47 (2009)

53. J.P. Bastard, M. Maachi, J.T. van Nhieu, et al, Adipose tissue IL-6 content correlates with resistance to insulin activation of glucose uptake both in vivo and in vitro, J. Clin. Endocrinol. Metab. 87, 2084-2089 (2002)

54. S. Glund, A. Deshmukh, Y.C. Long, et al, Interleukin-6 directly increases glucose metabolism in resting human skeletal muscle, Diabetes, 56, 1630-1637 (2007)

55. L. Dou, T. Zhao, L.L. Wang, et al, miR-200s Contribute to Interleukin-6 (IL-6)-induced Insulin Resistance in Hepatocytes, J. Biol. Chem. 31, 22596-22606 (2013)
56. Y.M. Niu, H. Yuan, Y.H. Liu, et al, The Effect of Adiponectin and AMPK in the Process of Aerobic Exercise Improving Insulin Resistance, Chin. J. Sports Med. 28, 36-40 (2009) 